\begin{tabular}{|c|l|}
\hline Title & Behaviour of liquid nitrogen between electrodes in a microgravity environment \\
\hline Author(s) & Suda, Y oshiyuki; Itoh, M.; Sakai, Y.; Matsuura, K.; Honma, N.; Kimura, T. \\
\hline Citation & $\begin{array}{l}\text { Cryogenics, 36/8), 567-571 } \\
\text { https://doi.org/10.1016/0011-2275(96)00054-9 }\end{array}$ \\
\hline Issue Date & 1996-08 \\
\hline Doc URL & http://hdl.handle.net/2115/10505 \\
\hline Type & article (author version) \\
\hline File Information & Y.Suda_Cryogenics1996_.pdf \\
\hline
\end{tabular}

Instructions for use 


\title{
Behaviour of liquid nitrogen between electrodes in a microgravity environment
}

\author{
Y. Suda, M. Itoh, Y. Sakai, K. Matsuura*, N. Honma* and T. Kimura** \\ Department of Electrical Engineering, Hokkaido University, Sapporo 060, Japan \\ *Department of Research and Development, Hokkaido Electric Power Corp., Sapporo 004, Japan \\ **Hokkaido Electric Power Corp., Asahikawa 078, Japan
}

Received 6 July 1995; revised 13 February 1996

\begin{abstract}
The motion of boiling liquid nitrogen $\left(\mathrm{LN}_{2}\right)$ between electrodes and its surface profile in a microgravity environment just after release from terrestrial gravity are observed. The dynamic behaviour is analysed considering the following forces: the Maxwell stress, surface tension and viscosity, and is explained consistently by theory including these forces. The velocity of the liquid driven by the Maxwell stress and the capillary force (surface tension) is compared with that driven by the capillary force only. The growth dynamics of bubbles produced on the surface of electrodes is discussed.
\end{abstract}

Keywords: liquid nitrogen; microgravity environment; Maxwell stress

In microgravity environments, interesting and important physical properties in fluids may appear, such as Marangoni convection $^{1}$; these are difficult to observe terrestrially because of the influence of gravity. An understanding of the physical phenomena of cryogenic liquids in microgravity environments is of practical importance for the development of planned superconducting systems in space. This motivates further study of the behaviour of coolants (e.g. liquid helium, nitrogen, hydrogen, etc.) in microgravity environments.

Recently, the possibility of controlling the shape of the meniscus and the velocity of the liquid/vapour phase interface of helium has been examined with electrostatic forces ${ }^{2,3}$. Important physical phenomena were identified in these studies, but a complete understanding of the phenomena has not yet been achieved. Studies on the capillary force of water in negligible gravity conditions ${ }^{4,5}$ and on capillary electrical phenomena and bubble growth in cryogenic liquids under the influence of gravity ${ }^{6,7}$ are important and helpful for understanding cryogenic liquid phenomena in space.

In this paper, we present the first observation of dynamic behaviour of boiling liquid nitrogen (LN,) between parallel plate electrodes just after release from terrestrial gravity and the structure of the liquid/gas interface meniscus between them in a microgravity environment. The behaviour of the liquid is analysed considering the influence of Maxwell stress, surface tension and viscosity, both with and without an electric field of $3 \mathrm{MV} \mathrm{m}^{-1}$. The growth in the liquid of bubbles produced on the surface of electrodes is also discussed. 


\section{Microgravity system}

The experiment was carried out in a free-fall capsule at the Japan Microgravity Center in Kamisunagawa, Hokkaido ${ }^{8}$. In this experiment, a microgravity environment of $<10^{-4} \mathrm{~g}$ ( $\mathrm{g}=$ acceleration due to terrestrial gravity) is sustained for $10 \mathrm{~s}$ in atmospheric pressure at room temperature. The dropshaft is $710 \mathrm{~m}$ below the ground. The capsule is composed of a thruster module, a payload module and a bass module as shown in Figure $1 a$. The $87 \mathrm{~cm} \mathrm{x} 87 \mathrm{~cm}$ x $90 \mathrm{~cm}$ free space available for the experiments is in the inner capsule. In the present experiment we use half this volume.

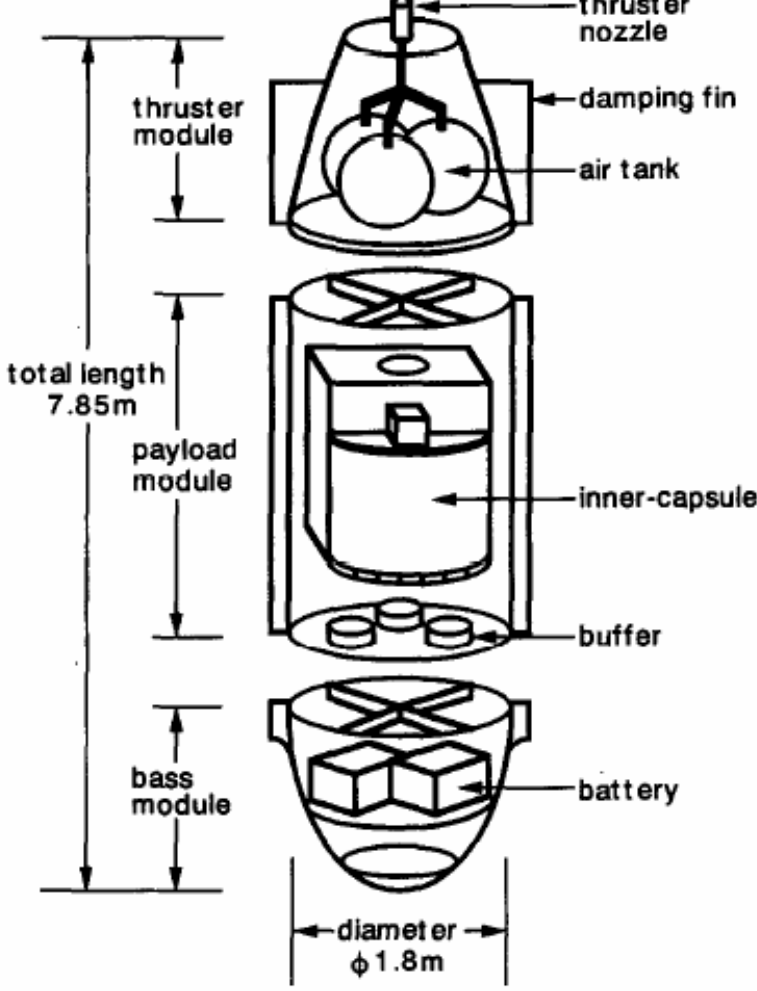

Figure 1 (a) Structure of free-fall capsule

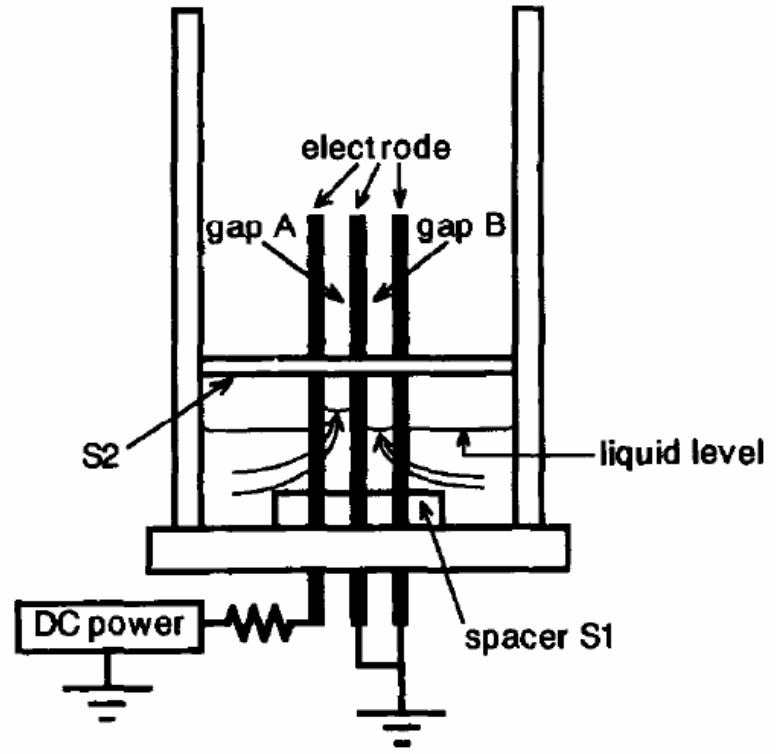

Figure 1 (b) Experimental cell

\section{Experimental set-up}

The experimental cell is shown in Figure $1 b$. Boiling $\mathrm{LN}_{2}$ and electrodes are contained in a glass vessel of $90 \mathrm{~mm}$ i.d. and $220 \mathrm{~mm}$ high. The vessel is thermally insulated, but the insulation is not sufficient to inhibit the generation of bubbles in the liquid because of the limited space. Three electrodes are set in the liquid. Across one pair (gap A), an electric field $E=3 \mathrm{MV} \mathrm{m}^{-1}$ is applied, and across the other (gap B) the electric field is zero. The inter-electrode distance $d$ for both gaps is $5 \mathrm{~mm}$. The electrode width $W$ is $10 \mathrm{~mm}$

Liquid motion between the electrodes is observed by back-illuminating the liquid column with a fluorescent lamp and recording the image using a CCD video camera at a rate of 30 frames s-i and an exposure time of $1 \mathrm{~ms}$.

The liquid level and the bubble size are measured on a video monitor, taking the following points into account. The picture of the image might be deformed due to the fact that the camera catches the light travelling through the curved 
glass vessel and that the camera cannot slide following the rise of the liquid surface, though the focus of the camera is at the centre of the gap. The deformation at the top of the electrodes consists of about $6 \%$ shrink in the vertical direction and about 3\% stretch in the horizontal direction. The liquid level is determined relative to the reference electrodes with a scale in the vessel. The liquid level and bubble volume data are calibrated accordingly. The image deformation for the video monitor display is around 3\%. The calibration for this deformation is not considered. Taking this error into account, the error in the present results is less than $5 \%$.

\section{Forces that act on the liquid}

\section{Maxwell stress, surface tension and viscosity}

Under the influence of terrestrial gravity, the forces that act on dielectric liquids under the present experimental conditions are the Maxwell stress $F_{\mathrm{M}}$, the surface tension $F_{\mathrm{S}}$, the force due to viscosity $F_{\mathrm{V}}$, and the force of gravity $\mathrm{mg}$ ( $\mathrm{m}=$ the mass of the liquid moving between the electrodes). Then, the velocity of a liquid $\mathrm{v}$ is determined by the following equation:

$m \mathrm{~d} v / \mathrm{d} t=F_{\mathrm{M}}+F_{\mathrm{S}}-F_{\mathrm{V}}-m g$

Here, in the parallel plate electrode configuration, $F_{\mathrm{M}}, F_{\mathrm{S}}$ and $F_{\mathrm{V}}$ may act in the plate direction and are given as follows:

$F_{\mathrm{M}}=\varepsilon_{0}\left(\varepsilon_{1}-\varepsilon_{\mathrm{g}}\right) E^{2} W d / 2$

$F_{\mathrm{S}}=2 \sigma W \cos \theta$

$F_{\mathrm{V}}=8 \mu v W h / d$

where $\varepsilon_{0}, \varepsilon_{1}, \varepsilon_{\mathrm{g}}, \sigma, \mu, \theta$ and $E$ are the permittivity of free space, the relative dielectric constants of the liquid and gaseous phase, the surface tension, viscosity of the liquid, the contact angle between the liquid and the electrode and the external field strength, respectively. Here, we take $0^{\circ}$ for $\theta$ taking account of the contact behaviour between the present stainless-steel electrode and $\mathrm{LN}_{2}$; $h$ is the length of the liquid column, which moves in the gap due to the forces.

The thermodynamic and electrical properties of nitrogen are listed in Table $1^{9}$.

The values of $h$ and $v$ after free fall is initiated $(t=0)$ are estimated by setting $g=0$ in Equation (1). Since the mass $m$ of the liquid which moves upwards in the gap, shown on the left-hand side of Equation (l), and $h$ and $v$ in Equation (4) are given as a function of time, Equation (1) has to be solved numerically. For the results presented here we have used a time step $\Delta t$ of $0.2 \mathrm{~ms}$ and the relationships $m(t)=\rho_{1} W d h(t), h(t)=h(t-\Delta t)+v(t-\Delta t) \Delta t$ and $v(t)=v(t-\Delta t)+$ $\alpha \Delta t$. Here $\alpha(=\mathrm{d} v / \mathrm{d} t)$ is the acceleration of the liquid, and the initial conditions are assumed to be $v=0$ and $h=h_{0}$ at $t$ $=0$. The value of $h_{0}$ is chosen to be $14 \mathrm{~mm}$ so as to obtain good agreement between theoretical and experimental values, since we cannot observe the bottom level from which liquid moves upwards in this electrode arrangement (see Figure 1b). 
Table 1 Thermodynamic and electrical properties of nitrogen at boiling point

Relative dielectric constant
Relative dielectric constant
Surface tension
Viscosity
Thermal conductivity
Latent heat of vaporization
Boiling temperature
Density of liquid phase
Density of gaseous phase
Isobaric specific heat

$$
\begin{aligned}
& \varepsilon_{\mathrm{l}}: 1.43 \\
& \varepsilon_{\mathrm{g}}: 1.00 \\
& \sigma: 8.8 \times 10^{-3} \mathrm{~J} \mathrm{~m}^{-2} \\
& \mu: 1.65 \times 10^{-4} \mathrm{~kg} \mathrm{~m}^{-1} \mathrm{~s}^{-1} \\
& \lambda: 0.14 \mathrm{~W} \mathrm{~m}^{-1} \mathrm{~K}^{-1} \\
& L: 199.1 \mathrm{~kJ} \mathrm{~kg}^{-1} \\
& T_{\mathrm{s}}: 77.348 \mathrm{~K}^{-3} \\
& \rho_{\mathrm{g}}: 804.2 \mathrm{~kg} \mathrm{~m}^{-3} \\
& \rho_{\mathrm{g}}: 4.59 \mathrm{~kg} \mathrm{~m}^{-3} \\
& C_{\mathrm{p}}: 2.03 \mathrm{~kJ} \mathrm{~kg}^{-1} \mathrm{~K}^{-1}
\end{aligned}
$$

\section{Growth of bubbles}

In liquids, the bubble radius $R$ expands with time $t$ as follows ${ }^{10}$ :

$R=2 J_{\mathrm{a}}(\lambda t / \pi)^{1 / 2}$, for $\zeta>>R$

or

$R=2\left(\zeta \mathrm{J}_{\mathrm{a}} / \pi\right)^{1 / 2}(\lambda t)^{1 / 4}$, for $\zeta<<R$

where $\zeta$ is the thickness of the superheated zone around a bubble. $J_{\mathrm{a}}$ and $\lambda$ are the Jacob's number and the thermal conductivity of a liquid, respectively. $J_{\mathrm{a}}$ is given as

$J_{\mathrm{a}}=C_{\mathrm{p}} \rho_{\mathrm{l}}\left(\Delta T_{\mathrm{s}} / L\right) / \rho_{\mathrm{g}}$

$\Delta T_{\mathrm{s}}=\left(\mathrm{l} / \rho_{\mathrm{g}}-\mathrm{l} / \rho_{\mathrm{l}}\right)\left(T_{\mathrm{s}} 2 \sigma L r_{0}\right)$

where $C_{\mathrm{p}}, \Delta T_{\mathrm{s}}, L, \rho_{\mathrm{g}}, \rho_{\mathrm{l}}, T_{\mathrm{s}}$ and $r_{0}$ are the isobaric specific heat, the degree of superheating (= $T-T_{\mathrm{s}}$ ), the latent heat of vaporization, the densities of gaseous and liquid nitrogen, the boiling temperature and the initial radius of a bubble, respectively. Here, effects of flow on $R$ can be neglected since the bubbles expand in the microgravity environment, as is clearly seen in the video pictures.

\section{Results and discussion}

Height of liquid surface between electrodes on the terrestrial globe

Figure 2 shows the liquid height $H$ in the gap determined from the background liquid surface. Values of $H$ may be 
determined to be 0 from the left-hand side of Equation (1). Since, $F_{\mathrm{V}}$ is 0 when the liquid is at rest and $F_{\mathrm{S}}$ is negligibly small, $H$ is found, by considering only the Maxwell stress and terrestrial gravity, to be

$H=\varepsilon_{0}\left(\varepsilon_{l}-\varepsilon_{\mathrm{g}}\right) E^{2} / 2 \rho_{\mathrm{l}} g$

Agreement between Equation (8) and our experiments showing an increase in $H$ in direct proportion to $E^{2}$ is an important benchmark validation of this experimental procedure.

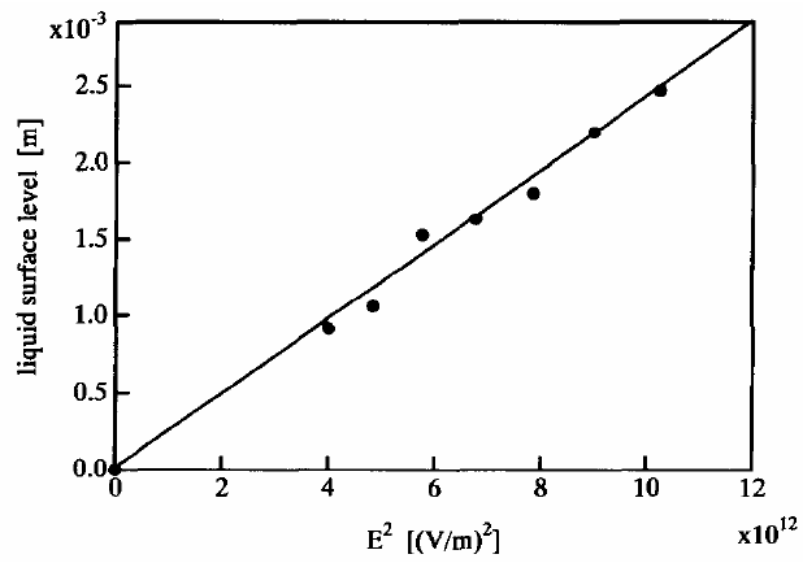

Figure 2 Rise in liquid level in gap as a function of electric field on terrestrial globe. $\bullet$, Experimental values; solid line, theoretical values

\section{Liquid motion under microgravity}

Pictures of liquid motion. Figure 3 shows pictures of the $\mathrm{LN}_{2}$ configuration taken with a CCD camera (a) just before microgravity starts, (b) $0.15 \mathrm{~s}$ after free fall has begun (microgravity initiation) and (c) $3 \mathrm{~s}$ after free fall has begun.

In Figure $3 a$ under terrestrial gravity, the raising of the liquid level in gap A $\left(E=3 \mathrm{MV} \mathrm{m}^{-1}\right)$ by Maxwell stress can be clearly identified; in contrast, surface tension is insufficient to result in a noticeable increase in the liquid level as seen in gap $\mathrm{B}(E=0)$.

In Figure $3 b$ at $0.15 \mathrm{~s}$, the liquid height and the meniscus of the liquid surface in gap A are clearly different from those in gap $B$. The liquid height in gap $A$ is significantly higher than that in gap $B$, since $F_{M}$ is around 4.7 times larger than $\boldsymbol{F}_{\mathrm{S}}$ for the present field strength. The liquid surface profile in gap A is clearly seen to be convex, while in gap $\mathrm{B}$ it is concave. The difference may be explained as follows. In the former case, $F_{\mathrm{M}}>F_{\mathrm{S}}$ leads to a velocity difference in the liquid inducing a viscous drag force at the electrode surfaces, and hence a convex surface. On the other hand, in gap B the capillary force draws the liquid up at the wall surface, which may result in the surface profile being concave. This is just the same as the laminar flow of a normal fluid in a pipe under a capillary force for contact angles $<90^{\circ}$, as described elsewhere ${ }^{4}$.

Figure $3 c$ shows that the liquid may be held stationary between two plates. The surface profile of the liquid at the top of gap A clearly differs from that of gap B. The former is convex because the electric field lines at the edge of the electrodes stray outwards; the liquid may be attracted along those lines. Therefore, although this surface profile is 
similar to that in gap A in Figure 3b, the mechanisms determining the profile shown in Figures $3 b$ and c are different. In gap B the liquid surface profile is determined by surface tension only, and accordingly it is concave, as seen in Figure 3c.

Many bubbles are seen in gap B, while in gap A only a few distorted bubbles are observed. Under terrestrial gravity, they would rapidly rise upward due to buoyancy and disappear, but here bubble growth can clearly be observed.
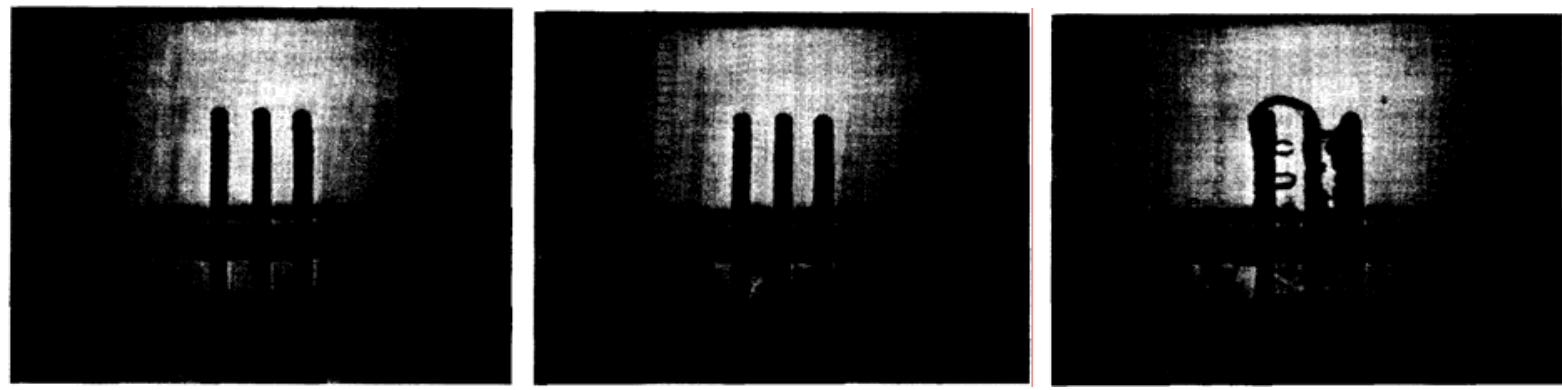

Figure 3 Pictures of liquid motion in gaps A and B, (a) just before microgravity starts, (b) $0.15 \mathrm{~s}$ after free fall has begun (microgravity initiation), (c) $3 \mathrm{~s}$ after free fall has begun

Motion of the liquid in the gaps. The experimentally measured values of the change in the liquid level from that on the terrestrial globe, as induced by the Maxwell stress and capillary force in gap A, are shown in Figure 4, along with theoretical values. They agree reasonably well with each other. In this trial only the initial stage of the liquid motion could be observed because of the limited length of the electrodes. The scatter of the experimental values may come from generation and growth of bubbles in the gap.

Liquid is drawn up the electrodes in gap B by capillary action, as shown in Figure 3. The change in the liquid surface level with time is presented in Figure 5. Data are not shown for the time period between 0.4 and $1.7 \mathrm{~s}$, since the surface could not be identified due to the electrode spacer S2 (see Figure $1 b$ ) being in front of the liquid and the probable eruption of bubbles under the surface during this period. Theoretical values agree reasonably well with the measurements for $t<0.4 \mathrm{~s}$. For $t>1.7 \mathrm{~s}$ theoretical values are not shown since initial values of the liquid level and velocity are not clear.

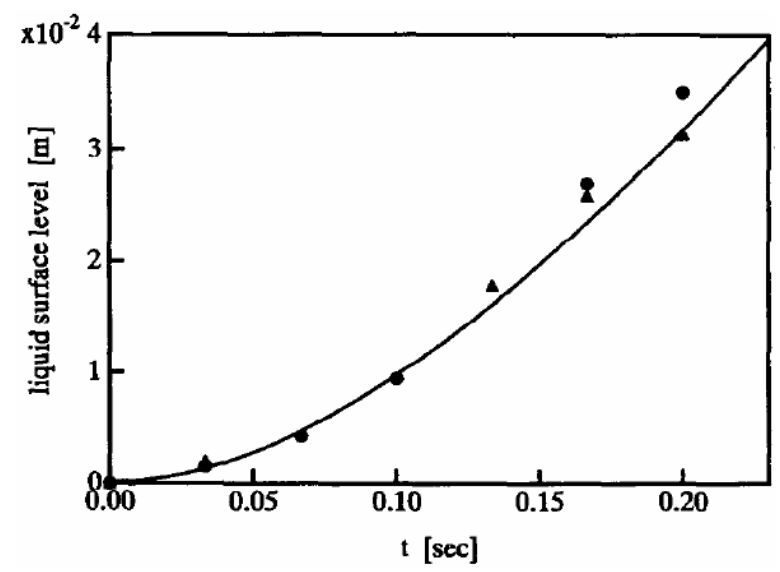

Figure 4 Change in liquid surface level between parallel plates in electric fields, showing experimental values at first trial $(\bullet)$ and at second trial $(\boldsymbol{\Delta})$. Solid line: theoretical values 


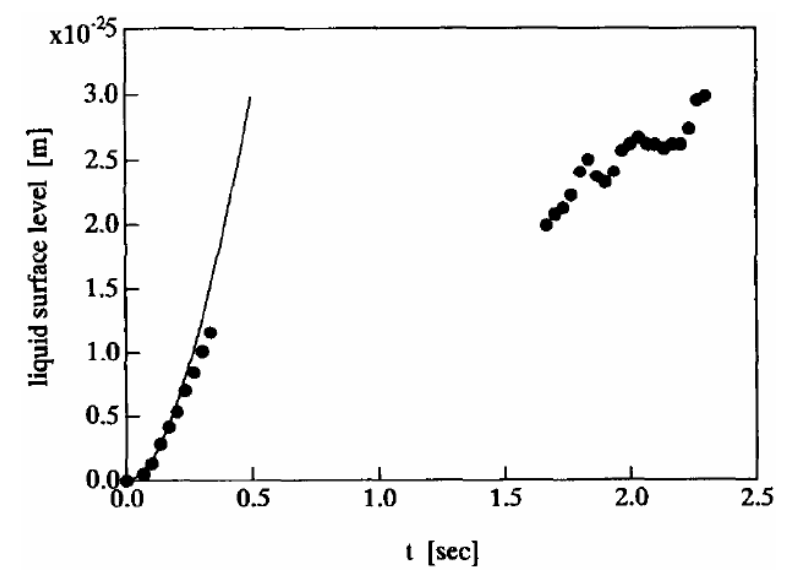

Figure 5 Change in liquid surface level between parallel plates without field. $\bullet$, Experimental values; solid line, theoretical values

These phenomena can be explained using the same arguments as described in previous studies ${ }^{4,5}$.

In this analysis, the liquid is supplied from the bottom sides of the electrodes in the vessel, as seen in Figure $1 b$, so the liquid just above the electrode spacer might not be moved. Therefore, the initial liquid column length ho from which the liquid moves upwards in the gap should be taken into account. Here, this is chosen to be $14 \mathrm{~mm}$ as a fitting parameter to describe the experimental values consistently. This value may be appropriate since the liquid level in the gap from spacer Sl (sse Figure 1 b) just before microgravity starts is about $20 \mathrm{~mm}$.

Growth of bubbles. Figure 6 shows bubble growth in boiling $\mathrm{LN}_{2}$ in gap A. These bubbles are generated by heat penetration from the free-fall capsule environment through the electrodes. It is generally reported that bubbles expand spherically and their radius increases with time according to Equations (5) or (6). However, in an electric field the bubbles grow ellipsoidally due to distortion by the Maxwell stress, as seen in Figure 3c; this is also observed in terrestrial gravity conditions ${ }^{11,12}$. Here, therefore, we will characterize the bubble by its volume instead of its radius.

Bubbles are generated at the surface of the high voltage electrode in a way similar to that described in a previous report ${ }^{11}$ and move towards the counter grounded electrode. The bubbles are clearly observed to grow at almost the same position as they were generated, since there is no buoyancy under microgravity conditions.

It is rather difficult to say conclusively from the present data whether the bubbles grow as $t^{3 / 2}$ or $t^{3 / 4}$ since the data lie between these functions. This may suggest that the superheated zone under these experimental conditions is ambiguous. Based on our experiment in which bubbles are generated by heat penetration, values of the initial radius of the bubble, $r_{0}$, the thickness of the superheated zone, $\zeta$, and the degree of superheating, $\Delta T_{\mathrm{S}}$, are estimated to be in the following ranges: $0.5 \mathrm{~mm}<r_{0},<1.1 \mathrm{~mm}, 0.8 \mathrm{~mm}<\zeta<1.2 \mathrm{~mm}$ and $1.2 \mathrm{mK}<\Delta T_{\mathrm{S}}<2.7 \mathrm{mK}$, respectively. These values are of the same order as those observed under the influence of terrestrial gravity ${ }^{11}$. Here we may conclude that differences in bubble growth in microgravity environments and on the terrestrial globe are not significant within the present experimental accuracy. 


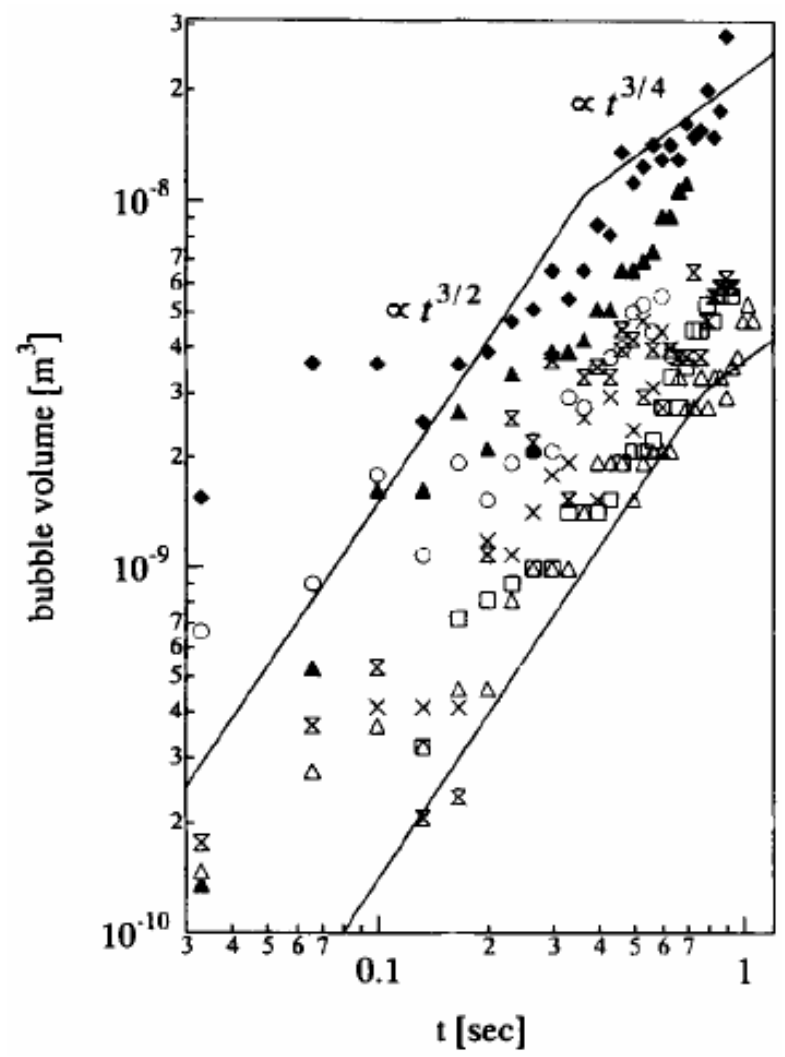

Figure 6 Increase in bubble volumes as a function of time. Symbols indicate volumes of different bubbles measured in the present experiment and solid lines the theoretical values calculated using Equations (5) and (6) with $r_{0}=0.5 \mathrm{~mm}, \zeta=1.2 \mathrm{~mm}, \Delta T_{\mathrm{S}}=2.7 \mathrm{mK}$, and $r_{0}=1.1 \mathrm{~mm}, \zeta=0.8 \mathrm{~mm}, \Delta T_{\mathrm{S}}=1.2 \mathrm{mK}$, respectively, thereby covering the experimental values

\section{Summary}

The dynamics of liquid nitrogen in a microgravity environment between parallel plate electrodes was observed, and explained by a force balance between the Maxwell stress, the surface tension and viscosity forces. Bubbles in the electrically stressed gap are distorted due to the Maxwell stress. Their growth process can be clearly observed because of the absence of a buoyancy force.

\section{Acknowledgements}

The authors would thank H. Tagashira, P. Ventzek and H. Sugawara for helpful discussion on dielectric liquid motion and the reviewers for insightful comments. 


\section{References}

1 Haga, M. et al. Effect of electric field on Marangoni convection under microgravity. J Jpn Soc Microgravity Appl (1995) 12 19-26

2 Mason, P.A. et al. The control of liquid helium in space by electrostatics for the satellite test of equivalent principle experiment Cryogenic Engineering Conference (1993) CD9

3 Israelsson, U.E., Jackson, H.W. and Petrac, D. Liquid/vapor phase separation in ${ }^{4}$ He using electric fields Cryogenics (1988) 28 120- 125

4 Ichikawa, N. and Satoda, Y. Interface dynamics of capillary flow in a tube under negligible gravity condition $J$ Colloid and Interface Sci (1994) 162 350-355

5 Dreyer, M., Delgado, A. and Rath, H.-J. Capillary rise of liquid between parallel plates under microgravity $J$ Colloid and Interface Sci (1994) 163 158-168

6 Hara, M., Kaneko, T., Yoshida, S. and Akazaki, M. Influence of the Sumoto effect on electrical breakdown in gap partially immersed in liquid nitrogen T IEE Japan (1984) 104-E 247-255

7 Hewitt, H.C. and Parker, J.D. Bubble growth and collapse in liquid nitrogen J Hear Transfer, Tram ASME, Series C (1968) 90 22-26

8 Kudoh, I. The world's largest underground dropshaft- Japan Microgravity Center J IEE Japan (1994) 114 781-784

9 Klipping, G. Lehrgang Handbuch Kryorechnik Uchida Rokakuho Pub. (1977) 26-27

10 Forster, K.E. Growth of a vapor-filled cavity near a heating surface and some related questions Phys Fluids (1961) 4 448-455

11 Hara, M., Ou, S., Saitou, H. and Matsumura, S. Thermal-bubble behaviour in liquid nitrogen under non-uniform fields T IEE Jpn (1993) 113-A 817-825

12 Beroual, A. Behavior of charged and uncharged bubbles in dielectric liquids subjected to electric stress $J$ Appl Phys (1992) 71 1142- 1145 